\title{
EQUATIONAL THEORIES OF ALGEBRAS WITH DISTRIBUTIVE CONGRUENCES
}

\author{
R. PADMANABHAN AND R. W. QUACKENBUSH ${ }^{1}$
}

ABSTRACT. If an equational class of algebras has the distributive or permutable congruence property then it is well known that it satisfies certain conditions, known as Mal'cev-type conditions. In this note such Mal'cev-type conditions are used to find minimal bases for certain equational theories of algebras. A typical result states that every finitely based equational theory of algebras with distributive and permutable congruences is one-based.

1. Let us start by recalling a result of B. Jónsson [2] that an equational class $\mathscr{K}$ of algebras has the distributive congruence property if and only if for some natural number $n \geqq 2 \mathscr{K}$ admits $n+1$ ternary polynomials $t_{0}, t_{1}, \cdots, t_{n}$ satisfying the identities

$$
\begin{aligned}
& t_{k}(x, y, x)=x \quad(k=0,1, \cdots, n), \\
& \Delta_{n}: \begin{array}{ll}
t_{k}(x, x, z)=t_{k+1}(x, x, z) & \text { if } k \text { is even, } \\
t_{k}(x, z, z)=t_{k+1}(x, z, z) & \text { if } k \text { is odd, }
\end{array} \\
& t_{0}(x, y, z)=x, \quad t_{n}(x, y, z)=z \text {. }
\end{aligned}
$$

For brevity, the conditions $\Delta_{n}$ are usually called " $n$-distributivity". The following result was first obtained by $\mathrm{K}$. Baker (Theorem 5.1 in [1]). We insert a quite elementary proof here.

LEMMA 1 (K. BAKER). Relative to $\Delta_{n}$, any finite set of identities can be equivalently expressed by a single identity.

Proof. As Baker has noted, in the presence of $\Delta_{n}$, any identity " $f=g$ " can be equivalently expressed by the $n-1$ identities

$$
t_{k}(u, f, v)=t_{k}(u, g, v), \quad k=1, \cdots, n-1 .
$$

Received by the editors March 12, 1973.

AMS (MOS) subject classifications (1970). Primary 08A25; Secondary 08A15, $20 \mathrm{~N} 15$.

Key words and phrases. Equational classes of algebras, equational theory, distributive congruence property, permutable congruence property, Mal'cev-type conditions, minimal bases.

${ }^{1}$ Research of both the authors was supported by the National Research Council of Canada.

(c) American Mathematical Society 1973 
Now, let $f_{i}\left(x_{i_{1}}, \cdots, x_{i_{n}}\right)=g_{i}\left(x_{i_{1}}, \cdots, x_{i_{n}}\right), i=1, \cdots, m$, be any set of $m$ identities.

Let $\Sigma=\left\{t_{k}\left(u_{k}, f_{i}, v_{k}\right)=t_{k}\left(u_{k}, g_{i}, v_{k}\right) \mid 1 \leqq i \leqq m, 1 \leqq k \leqq n-1\right\}$. Abbreviate $t_{k}\left(u_{k}, f_{i}, v_{k}\right)$ by $a_{i_{k}}$ and $t_{k}\left(u_{k}, g_{i}, v_{k}\right)$ by $b_{i_{k}}$. Relative to $\Delta_{n}, \Sigma$ has two properties: (i) $\Sigma$ holds iff the identities $f_{i}=g_{i}$ hold for all $i=1, \cdots, m$; (ii) each polynomial $a_{i_{k}}, b_{i_{k}}$ is idempotent (i.e.)

$$
a_{i_{k}}(x, \cdots, x)=b_{i_{k}}(x, \cdots, x)=x .
$$

Hence, $\Sigma$ can be equivalently expressed by a single identity, relative to $\Delta_{n}$. Let us illustrate this with two identities (which is sufficient): let

$$
a_{1}\left(x_{1}, \cdots, x_{n}\right), \quad b_{1}\left(x_{1}, \cdots, x_{n}\right), \quad a_{2}\left(y_{1}, \cdots, y_{m}\right), \quad b_{2}\left(y_{1}, \cdots, y_{m}\right)
$$

be polynomials such that

$$
\Delta_{n} \vDash a_{1}(x, \cdots, x)=b_{1}(x, \cdots, x)=a_{2}(x, \cdots, x)=b_{2}(x, \cdots, x)=x \text {. }
$$

Then the set of two identities $a_{1}\left(x_{1}, \cdots, x_{n}\right)=b_{1}\left(x_{1}, \cdots, x_{n}\right)$ and $a_{2}\left(y_{1}, \cdots, y_{m}\right)=b_{2}\left(y_{1}, \cdots, y_{m}\right)$ is clearly equivalent to the identity

$$
\begin{aligned}
a_{1}\left(a_{2}\left(y_{11}, \cdots, y_{1 m}\right), a_{2}\left(y_{21}, \cdots, y_{2 m}\right), \cdots, a_{2}\left(y_{n 1}, \cdots, y_{n m}\right)\right) \\
\quad=b_{1}\left(b_{2}\left(y_{11}, \cdots, y_{1 m}\right), b_{2}\left(y_{21}, \cdots, y_{2 m}\right), \cdots, b_{2}\left(y_{n 1}, \cdots, y_{n m}\right)\right) .
\end{aligned}
$$

This completes the proof of the lemma.

LEMMA 2. If $n>2$ then the set of identities $\Delta_{n}$ is equivalent to a set of $n-1$ identities.

Proof. The language of $\Delta_{n}$ consists of $n-1$ ternary operations $t_{1}, t_{2}, \cdots, t_{n-1}\left(t_{0}\right.$ and $t_{n}$ were introduced so that the expressions $\Delta_{n}$ become elegant) and each $t_{i}$ is idempotent. Hence the theory of $\Delta_{n}$ is an equational theory of idempotent algebras of type $\langle 3,3, \cdots, 3\rangle(n-1$ times) and hence, by [6], is $n-1$ based.

From Lemmas 1 and 2, we get the

COROLlaRY 1. If $n>2$ then every finitely based equational class of algebras with the n-distributivity property is $n$-based.

When $n=2, \Delta_{n}$ consists of just one ternary polynomial $t(x, y, z)$ satisfying the identities

$$
t(x, y, x)=t(x, x, z)=t(z, x, x)=x ;
$$

i.e. " $t$ " is a majority polynomial.

LEMMA 3. A ternary polynomial symbol " $t$ " is a majority polynomial iff it satisfies the identity

$$
t(t(x, y, y), u, t(t(x, y, y), y, z))=y .
$$


Proof. The "only if" part being obvious we need prove only the "if" part.

Putting $z=t(t(x, y, y), y, w)$ in (1) we get

$$
t(t(x, y, y), y, z)=t(t(x, y, y), y, t(t(x, y, y), y, w))=y
$$

by (1) and hence (1) reduces to

$$
t(t(x, y, y), u, y)=y .
$$

Putting $x=t(a, y, y)$ in (2) we notice that the term $t(x, y, y)$ becomes $t(t(a, y, y), y, y)$ which is simply " $y$ " by (2) and hence we get

$$
t(y, u, y)=y .
$$

Now put $z=t(x, y, y)$ in (1). By two successive applications of (3) we have

$$
t(x, y, y)=y
$$

and hence (1) reduces to $t(y, u, t(y, y, z))=y$. Finally the substitution $u=t(y, y, z)$ and an application of (4) yields the last of the majority conditions $t(y, y, z)=y$, and this completes the proof of the lemma.

Combining this result with Lemma 1 along with Corollary 1 we get the following

THEOREM 1. A finitely based equational class of algebras with the $n$ distributive congruence property is $n$-based.

REMARK. If $n=2$ the above result is the best possible, since, for example, the theory of distributive lattices is not one-based (see [5]). However, it is not known whether the above is the best possible in other cases.

2. If an equational class $\mathscr{K}$ of algebras has both the distributive congruence property and the permutable congruence property then A. F. Pixley ([7], [8]) has shown that $\mathscr{K}$ admits a ternary polynomial $p$ satisfying

$$
p(y, y, x)=p(x, z, z)=p(x, u, x)=x
$$

and conversely.

Let $\mathscr{K}$ be an equational class of algebras with distributive and permutable congruences. Of course, by Lemma 1 , it follows that, in the presence of the identities (5), the validity of any finite number of identities is equivalent to that of a single one. However, the identities (5) are so nice that this becomes an easy verification. Indeed, in presence of (5), any identity $f\left(x_{1}, \cdots, x_{n}\right)=g\left(x_{1}, \cdots, x_{n}\right)$ is equivalent to the identity 
$p(u, f, g)=u$. Now, let

$$
f\left(y_{1}, \cdots, y_{n}\right)=y_{1}, \quad g\left(z_{1}, \cdots, z_{m}\right)=z_{1},
$$

be two identities. Consider the identity

$$
p\left(x, f, y_{1}\right)=p\left(x, g, z_{1}\right) \text {. }
$$

Clearly (6) implies (7). Conversely, identifying $x=y_{1}=g\left(z_{1}, \cdots, z_{m}\right)$ in (7) and using the first and the last of the identities (5) we get $g\left(z_{1}, \cdots, z_{m}\right)=$ $z_{1}$ and similarly we have $f\left(y_{1}, \cdots, y_{n}\right)=y_{1}$.

THEOREM 2. Every finitely based equational theory of algebras with distributive and permutable congruences is one-based.

Proof. Thus it is sufficient if we show that these four identities (5) and $f\left(z, z_{1}, \cdots, z_{n}\right)=z$ can be equivalently expressed by a single identity. Now consider

$$
p(p(u, u, x), p(f, y, z), z)=x .
$$

Certainly the four identities with which we started jointly imply (8). Thus it remains to show that (8) implies the four identities.

Define $f_{1} \equiv f\left(x, z_{1}, \cdots, z_{n}\right)$ and put $u=p\left(f_{1}, f_{1}, x\right)$ in (8). Then

$$
p(u, u, x)=p\left(p\left(f_{1}, f_{1}, x\right), p\left(f_{1}, f_{1}, x\right), x\right)=x
$$

by $(8)$ and hence $(8)$ reduces to

$$
p(x, p(f, y, z), z)=x .
$$

Thus, by (8) and (9) we have

$$
p(u, u, x)=x .
$$

Now the substitution $x=p(f, y, z)$ in (9) along with one application of (10) yields

$$
p(f, y, z)=z
$$

By (9) and (11) we have

$$
p(x, z, z)=x \text {. }
$$

The substitution $y=z$ in (11) along with (12) yields the identity

$$
f\left(z, z_{1}, \cdots, z_{n}\right)=z
$$

and finally by (11) and the above we have $p(z, y, z)=z$ and this completes the proof of the theorem.

Baker has shown that every finite algebra of an arbitrary equational class with distributive congruences has a finite base (see also M. Makkai, 
Notices Amer. Math. Soc. 20 (1972), p. A-254). This, coupled with our Theorem 2, yields the following

COROLLARY 2. Every finite algebra of an equational class with distributive and permutable congruences is one-based.

For example, all quasi-primal algebras are such (see e.g. [8]) and hence are one-based. For primal algebras, this result was previously obtained by G. Grätzer and R. McKenzie [3]. As stated before, Corollary 2 is not true once we drop the permutable congruence property from the hypothesis. On the other hand, T. C. Green [4] has shown that, given $n$, there are finitely based equational theories with permutable congruences (in fact, definitionally equivalent to groups) which are $n$-based but not $n-1$ based. This shows that the distributive congruence property cannot be dropped either.

\section{REFERENCES}

1. Kirby A. Baker, Primitive satisfaction and equational problems for lattices and other algebras (preprint).

2. B. Jónsson, Algebras whose congruence lattices are distributive, Math. Scand. 21 (1967), 110-121. MR 38 \#5689.

3. G. Gratzer and R. McKenzie, Equational spectra and reduction of identities, Notices Amer. Math. Soc. 14 (1967), 697. Abstract \#67T-469.

4. Thomas C. Green, Minimum bases for equational theories of groups and rings (preprint).

5. R. McKenzie, Equational bases for lattice theories, Math. Scand. 27 (1970), 24-38. MR 43 \#118.

6. R. Padmanabhan, Equational theory of idempotent algebras, Algebra Universalis 2 (1972), 57-61.

7. A. F. Pixley, Distributivity and permutability of congruence relations in equational classes of algebras, Proc. Amer. Math. Soc. 14 (1963), 105-109. MR 26 \#3630.

8. 는 The ternary discriminator function in universal aigebra, Math. Ann. 191 (1971), 167-180. MR 45 \#1820.

Department of Mathematics, University of Manitoba, Winnipeg, Manitoba R3T 2N2, CANADA 